\title{
Structure and Dynamics of Supercoil-Stabilized DNA Cruciforms
}

\author{
Luda S. Shlyakhtenko', Vladimir N. Potaman², Richard R. Sinden² \\ and Yuri L. Lyubchenko'*
}

${ }^{1}$ Departments of Biology and Microbiology, Arizona State University, Tempe AZ 85287-2701, USA

${ }^{2}$ Institute for Bioscience and Technology, Department of Biochemistry and Biophysics Texas AEM University Houston, TX 77030, USA

\begin{abstract}
Understanding DNA function requires knowledge of the structure of local, sequence-dependent conformations that can be dramatically different from the $B$-form helix. One alternative DNA conformation is the cruciform, which has been shown to have a critical role in the initiation of DNA replication and the regulation of transcription in certain systems. In addition, cruciforms provide a model system for structural studies of Holliday junctions, intermediates in homologous DNA recombination. Cruciforms are not thermodynamically stable in linear DNA due to branch point migration, which makes their study using many biophysical techniques problematic. Atomic Force Microscopy (AFM) was applied to visualize cruciforms in negatively supercoiled plasmid DNA. Cruciforms are seen as clear-cut extrusions on the DNA filament with the lengths of the arms consistent with the size of the hairpins expected from a $106 \mathrm{bp}$ inverted repeat. The cruciform exists in two different conformations, an extended one with the angle of ca. $180^{\circ}$ between the hairpin arms and a compact, X-type conformation, with acute angles between the hairpin arms and the main DNA strands. The ratio of molecules with the different conformations of cruciforms depends on ionic conditions. In the presence of high salt or $\mathrm{Mg}$ cations, a compact, X-type conformation is highly preferable. Remarkably, the X-conformation was highly mobile allowing the cruciform arms to adopt a parallel orientation. The structure observed is consistent with a model of the Holliday junction with a parallel orientation of the exchanging strands.
\end{abstract}

(C) 1998 Academic Press

Keywords: inverted repeats in DNA; cruciform conformation; DNA supercoiling; Holliday junction; Atomic Force Microscopy

\section{Introduction}

Local sequence-dependent DNA conformations can be dramatically different from the canonical $B$-form helix. One class of alternative DNA conformations is cruciforms, which can form in DNA sequences with a 2-fold symmetry (inverted repeats or palindromes; Gierer, 1966). Cruciform formation requires negative DNA supercoiling and sufficient supercoiling exists in cells that cruciforms can exist in vivo (reviewed by Sinden, 1994). Inverted repeats are present in many functionally important genome regions. Specifically, inverted repeats are associated with origins of replication in plasmids and in many prokaryotic and eukaryotic

Abbreviations used: AFM, Atomic Force Microscopy; AP-mica, mica functionalized with

3-aminopropyltriethoxy silane. viruses (Kornberg \& Baker, 1992). An involvement of cruciform in the regulation of gene expression has been suggested based on the requirement of inverted repeat symmetry for transcription of some genes (Glucksmann et al., 1992). Another motivation in the study of cruciforms is their putative equivalence to Holliday junctions (Lilley \& Kemper, 1984). The latter type of DNA structure is an intermediate in a homologous DNA recombination (Sigal \& Alberts, 1972; Kowalczykowski, 1994), and the cruciform structure might be the most appropriate system for modeling Holliday junctions.

Inverted repeats under physiological conditions can adopt cruciform structures which are stabilized by unrestrained negative DNA supercoiling (reviewed by Sinden, 1994). The formation of open regions at the loops and possible base unstacking at the junction make the existence of cruciforms in 
linear DNA thermodynamically less favorable (reviewed by Vologodskii, 1992), and they are never observed in stable form in linear DNA molecules. The details of the secondary structure of cruciforms have been elucidated utilizing enzymatic and chemical techniques. Several bases at the loops are easily accessible to chemical probes and to single strand-specific enzymes, indicative of unpaired and unstacked bases (Sinden, 1994; Palecek, 1992). The bases at the junction are much less reactive, but they can be attacked by chemical probes at elevated temperatures or under conditions of low salt (McClellan \& Lilley, 1987; Voloshin et al., 1989). Several studies using different physical chemical techniques have analyzed the structure of stable, immobile four-way DNA junctions (Cooper \& Hagerman, 1987; Lilley \& Clegg, 1993; Seeman \& Kallenbach, 1994; Lilley, 1997). However, there has been little attempt to analyze the spatial geometry of cruciforms. In large part, this is due to the instability of cruciforms in small linear DNA molecules. Cruciforms are not thermodynamically stable in linear DNA due to branch point migration. Moreover, stable cruciforms in relatively large negatively supercoiled DNA molecules are not amenable to studies by traditional structural biology methods, such as high resolution NMR and crystallography. Electron microscopy has been used to visualize cruciform structures, but this system involved totally palindromic supercoiled circular DNA, in which the size of cruciforms varied considerably ( Mizuuchi et al., 1982).

Since the early 1990s another visualization technique, Atomic Force Microscopy (AFM; Binnig et al., 1986), has become available for imaging DNA and nucleoprotein complexes (Bustamante et al., 1994; Hansma \& Hoh, 1994; Lyubchenko et al., 1995). We have developed a sample preparation method permitting the deposition and imaging of the sample in a broad range of environmental conditions (Lyubchenko et al., 1992, 1993a,b, 1995). This method has been recently applied to structural studies of supercoiled DNA (Lyubchenko \& Shlyakhtenko, 1997) to demonstrate directly that the overall structure of supercoiled DNA dramatically depends on ionic conditions. Here we report a visualization of cruciforms in supercoiled DNA. The AFM data demonstrate directly and unambiguously two different cruciform conformations, a compact X-type and an extended one. The capability of AFM to operate in aqueous solutions allowed us to detect a surprisingly high conformational flexibility of cruciforms. In particular, an X-type conformation was very mobile, allowing the hairpin arms to move between the orthogonal and almost parallel orientations. The geometry of cruciforms with parallel hairpin arms is structurally equivalent to Holliday junctions with a parallel orientation of exchanging strands.

\section{Results}

\section{AFM imaging of cruciforms in supercoiled DNA}

We used plasmid pUC8F14C DNA containing a 106-bp F14C inverted repeat with an expected cruciform arm length of $53 \mathrm{bp}$ (Sinden, 1994; Kochel \& Sinden, 1988). We prepared DNA samples with different superhelical densities including those sufficient for the cruciform transition in all of the topoisomers (Kochel \& Sinden, 1988). The key in the AFM sample preparation procedure is the use of the functionalized substrate (AP-mica) which permits one to obtain high resolution AFM images of DNA by a one step deposition from a very wide range of ionic conditions (Lyubchenko et al., 1992, 1993a,b; Lyubchenko \& Shlyakhtenko, 1997). This procedure is highly reproducible and allows one to perform imaging in air or in aqueous solutions (Lyubchenko et al., 1992, 1993b; Lyubchenko \& Shlyakhtenko, 1997).

The AFM images of the sample prepared by deposition from a low salt solution (TE buffer) are shown in Figure 1. A fraction of DNA topoisomers with two to six supercoils remaining after the transition $\left(\left(L k-L k_{0}\right)=-12\right.$ to -18$)$ was used in the experiments shown in Figure 1A. The main feature of these images is the presence of rather long extrusions indicated by arrows. These extrusions are generally of two types; those with either extended or acute geometry of cruciform arms. In the extended geometry, both arms lie almost in one line, so that the angle between them is ca. 180 (molecules 2 and 4). Extrusions of this type were found in $25( \pm 5) \%$ of molecules. Molecules in which the cruciform arms form a rather acute angle (X-type geometry, molecules 1, 3, 5, 6) with the DNA strand being sharply bent were the most common, ca. $75( \pm 5) \%$. Zoomed images of the molecules with linear extrusions and those with the X-type geometry are shown as insets (i) and (ii), respectively. The size of the arms measured for extended extrusions is 15 to $20 \mathrm{~nm}$, in full agreement with the expected length of the cruciform hairpins containing $53 \mathrm{bp}$ (18 $\mathrm{nm}$ for $B$-helix DNA geometry).

At lower superhelical density $\left(\left(L k-L k_{0}\right)=-7\right.$ to $-13)$, molecules that appeared to be relaxed circles with extrusions in extended and X-type conformations were observed; two examples are shown in Figure $1 \mathrm{~B}$ and $\mathrm{C}$, respectively. According to two-dimensional gel electrophoresis data, the transition of the 106-bp inverted repeat into the cruciform structure takes place in a very narrow range of $\left(L k-L k_{0}\right)$ (from -9 to -10$)$, so the cruciform-containing plasmids of this sample should be topologically relaxed molecules. Crucforms will persist in these molecules, since cruciforms are stable in DNA with very low superhelical density (Sinden et al., 1983). The AFM observations of relaxed DNA molecules containing cruciforms agree with electrophoretic data, which support the hypothesis that the extrusions visualized with 

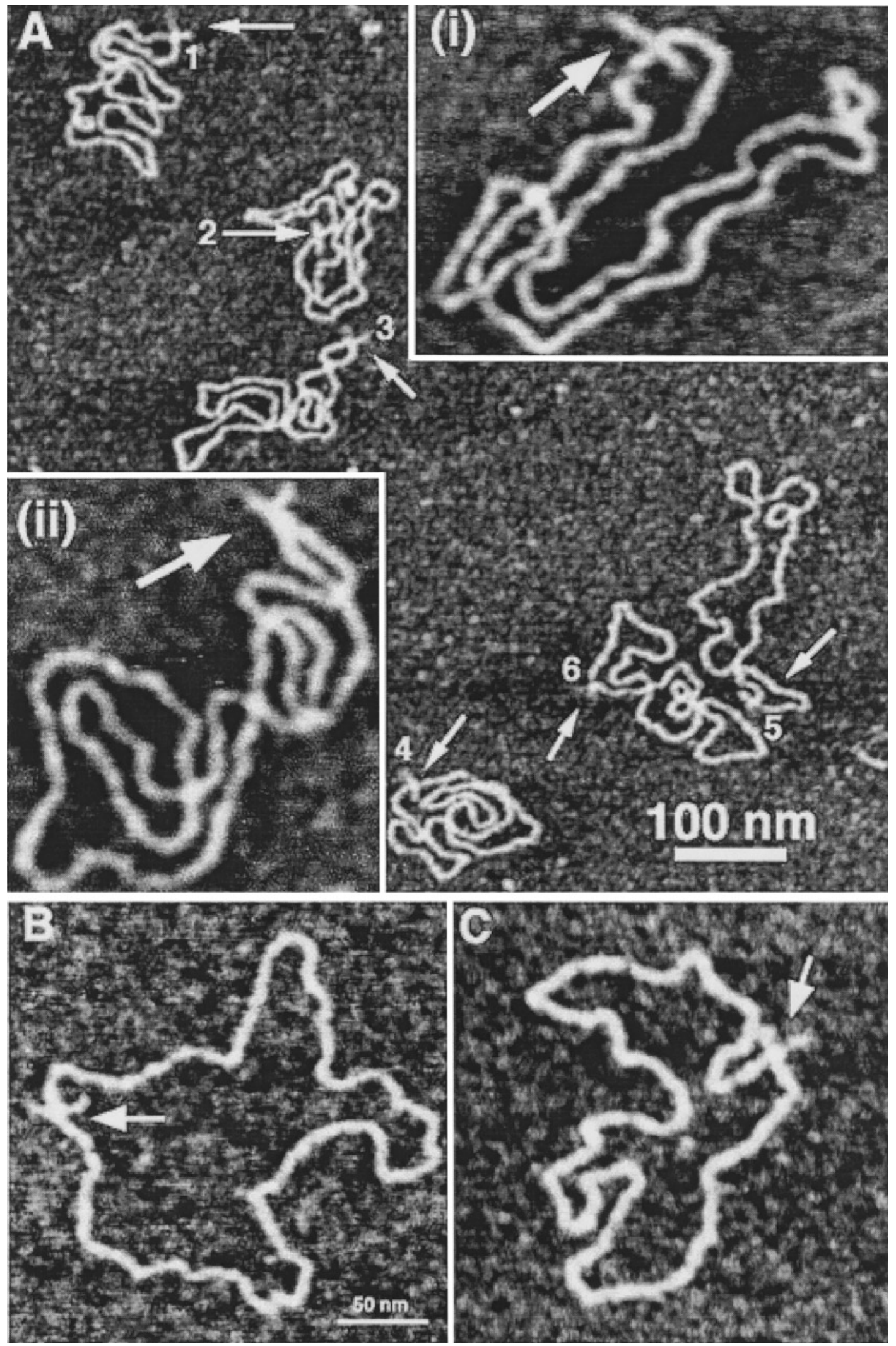

Figure 1. AFM images of pUC8F14C plasmid DNA obtained by imaging in air. The cruciforms are indicated with arrows and numbered. A, For these studies a sample, which contained a set of topoisomers with the linking number difference $L k$ from -12 to -18 , was used. The insets (i) and (ii) are the zoomed images of extended and X-type conformations of the cruciforms, respectively. B and C, These images were obtained for the sample with lower supercoiling density $\left(\left(L k-L k_{0}\right)\right.$ from -7 to -13$)$. 
AFM are, in fact, the cruciform arms of the $106 \mathrm{bp}$ palindrome.

\section{Effect of ionic conditions on conformation of the cruciform}

To study the effect of ionic strength on the cruciform conformation, DNA was deposited from high salt solutions (TE buffer plus $200 \mathrm{mM} \mathrm{NaCl}$ ). The data for the sample with $\left(L k-L k_{0}\right)=-12$ to -18 are shown in Figure 2A, where unambiguously identified cruciforms are indicated with arrows. A small scale image with three DNA molecules is shown in the inset. Under these high ionic conditions the cruciform conformation was predominantly the X-type. Only a few DNA molecules contained cruciforms in the extended conformation (e.g. molecule 2). The data for a highly supercoiled DNA sample $\left(\left(L k-L k_{0}\right)=-18\right.$ to -24$)$ also indicate that the conformation of the cruciform is mainly X-type (Figure 2B). Similar results were obtained for the sample prepared in TE buffer plus $10 \mathrm{mM} \mathrm{MgCl}$. Thus, cations stabilize the X-type cruciform structure rather than an extended one. These results are generally consistent with the effect of cations on geometry of immobile four-way junctions (reviewed by Cooper \& Hagerman, 1987; Lilley \& Clegg, 1993; Seeman \& Kallenbach, 1994; Lilley, 1997): the junctions change from an extended conformation into the X-type geometry upon increasing concentrations of $\mathrm{Mg}$ or $\mathrm{Na}$ ions.

To compare the geometric characteristics of the X-type cruciform at different ionic conditions, we measured the angles between the hairpins and between the main DNA strands for the images obtained under low salt conditions (TE buffer), high salt conditions $(200 \mathrm{mM} \mathrm{NaCl})$, and in the presence of $\mathrm{Mg}$ cations. The data in Table 1 show that there was only a very weak effect of ionic conditions on the X-type geometry of the cruciform: the mean values for the angles are rather insensitive to a high ionic strength or the presence of $\mathrm{Mg}$ cations. A slightly higher value for the angle between the main strand DNA arms under low salt conditions may be due to a more extended conformation of supercoiled DNA in low salt solutions as found in our earlier work (Lyubchenko \& Shlyakhtenko, 1997). In addition, there is a very high variability of the inter-arm angles (see, for example, molecules 2 and 3 in Figure 2B). These angles in different molecules vary in such a broad range that the molecules with almost parallel orientation of the hairpin arms are present in a high proportion. These data suggest that the arms of the cruciform move in a rather broad range, so the high variability of the angles between the hairpin arms may represent snapshots of a highly mobile structure. To observe the dynamics of cruciforms directly, we performed AFM imaging in solution omitting the step of sample drying (AFM in situ; Lyubchenko \& Shlyakhtenko, 1997). In this case, DNA molecules are loosely attached to the surface of a weakly cationic AP-mica, that allows DNA fragments between anchoring points to retain a quasi-solution mobility (Lyubchenko \& Shlyakhtenko, 1997).

\section{The direct observation of dynamics of the cruciform}

The DNA sample was prepared in TE buffer, because at these ionic conditions we observed both
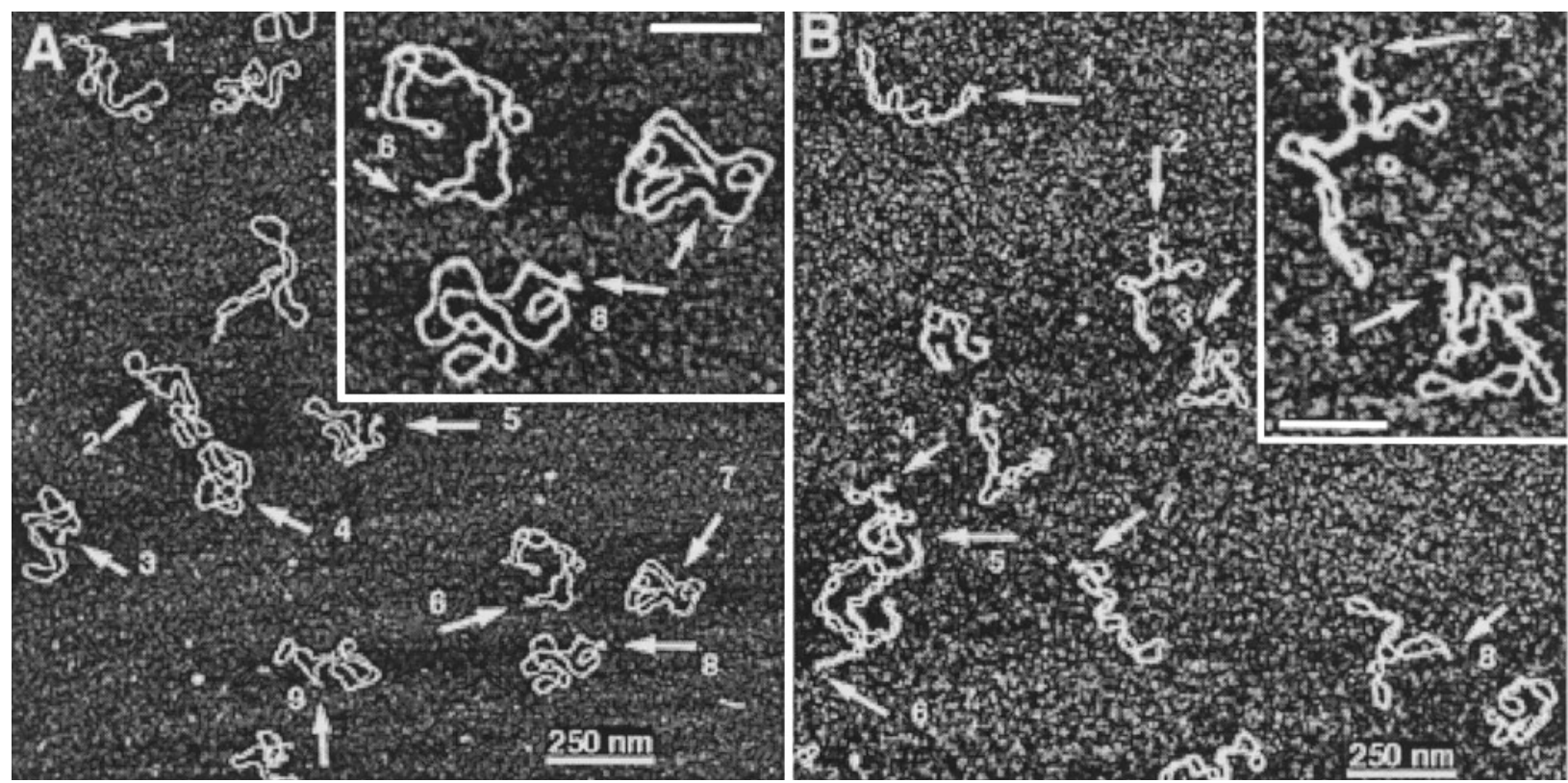

Figure 2. AFM images of the samples deposited at high ionic conditions (TE buffer plus $200 \mathrm{mM} \mathrm{NaCl}$ ). Images in A were obtained for the sample with $\left(L k-L k_{0}\right)$ from -12 to -18 . AFM data for highly supercoiled sample $\left(\left(L k-L k_{0}\right)\right.$ from -18 to -24$)$ are shown in B. The cruciforms are indicated with arrows. The scale bar for the insets represents $125 \mathrm{~nm}$. 
Table 1. The angles between the hairpin arms and the main DNA strands of the cruciform in X-type conformation

\begin{tabular}{lccc}
\hline & $\begin{array}{c}\text { The angle between the } \\
\text { hairpin arms } \\
\text { (degrees } \pm \text { SD) }\end{array}$ & $\begin{array}{c}\text { The angle between the } \\
\text { main strands } \\
\text { (degrees } \pm \text { SD) }\end{array}$ & $\begin{array}{c}\text { The number of } \\
\text { molecules } \\
\text { measured }\end{array}$ \\
\hline TE-buffer & $71 \pm 36$ & $83 \pm 38$ & 38 \\
TE-buffer plus 10 $\mathrm{mM} \mathrm{MgCl}_{2}$ & $74 \pm 38$ & $66 \pm 34$ & 32 \\
TE buffer plus $200 \mathrm{mM} \mathrm{NaCl}$ & $67 \pm 37$ & $59 \pm 40$ & 33 \\
\hline
\end{tabular}

the extended and X-type geometry cruciform conformation. DNA solution was injected into the imaging cell and the images of the molecules bound to the surface were taken (Figure 3). The images A and $B$ are six scans from a series of scans over the same area with three DNA molecules containing cruciforms of different geometries. Molecule 1 (Figure 3A) contains the cruciform in an extended conformation which remained unchanged in the next image (Figure 3B). The cruciform conformation of molecule 2 is of the X-type with the ca. $70^{\circ}$ angle between the arms (Figure 3A). Upon rescanning, however, the arms dramatically changed their orientation becoming almost parallel to each other as shown in Figure 3B. The data obtained for six consecutive scans are shown in Figure 3C. Each of the three zoomed regions of the initial images shows the dynamics of one specific cruciform in individual columns. The images in the first column show that the extended conformation of cruciform 1 did not change during the entire observation period. On the contrary, the X-type conformation of the cruciform 2 was very mobile. The hairpin arms of cruciform 2 were initially quite far away from each other (with an angle of ca. 100), but then they moved (frames c and d) and finally adopted a parallel orientation (frame f). The behavior of cruciform 3 was quite different. Initially, the angle between the hairpins was ca. $60^{\circ}$, then the arms became parallel (frames $c$ and $d$ ) and moved apart again at the end of the experiment (frames e and f). Thus, the X-type structure of cruciforms is very mobile, which explains a large variability of the angle values shown in Table 1. A relatively low mobility of the main DNA strands in experiments in liquid can be explained by their stronger binding to the surface compared with the short hairpin arms. Unlike the X-type conformation, the extended cruciform geometry is less dynamic. We measured the angles between the hairpins and the main DNA strands. The statistics for the opposite (vertical) acute angles, $76( \pm 11)$ and $79( \pm 10)$, indicate that the hairpin arms of extended cruciforms have a very low mobility. This conclusion is consistent with the AFM in situ data (Figure 3C) showing that cruciform 1 did not change its shape during the entire observation period.

\section{Discussion}

\section{The geometry of the cruciforms on the surface and in solution}

The results we obtained demonstrate clearly that a local alternative DNA structure, such as the cruciform, can be visualized with AFM. The cruciform arms can lie on opposite sides of the main strand which is almost straight in the vicinity of
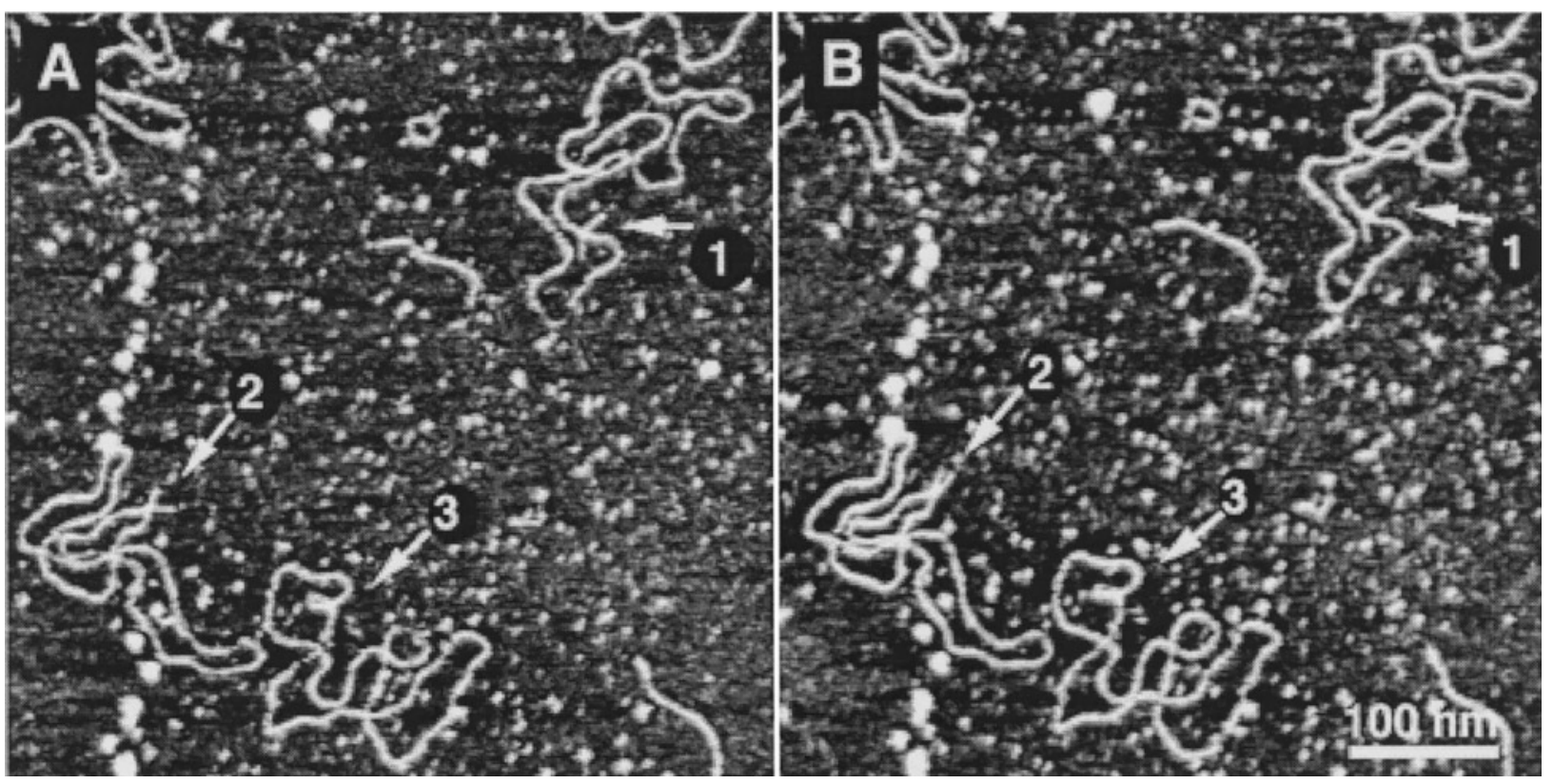

Figure 3(A and B) (legend on page 66) 


\section{C}

Cruciform 1
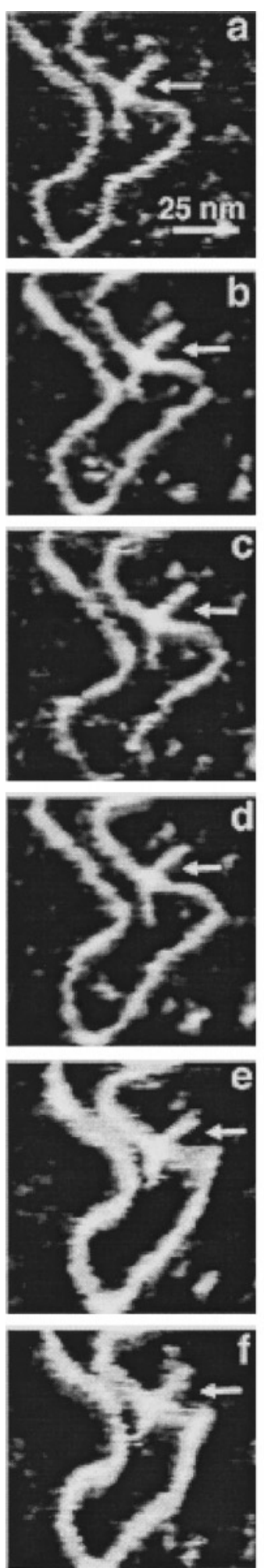

Cruciform 2
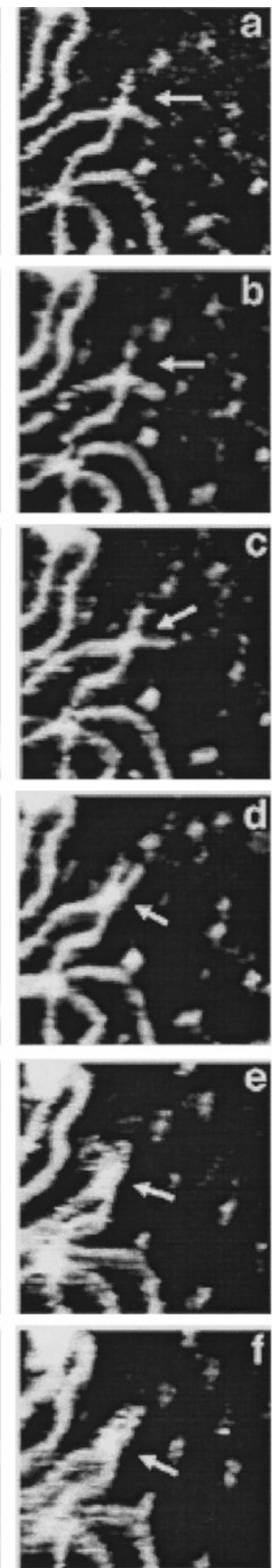

Cruciform 3
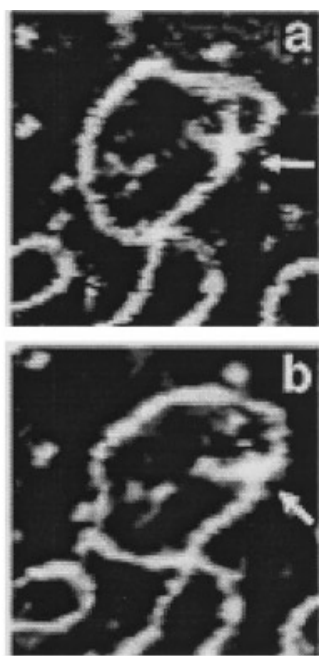

$5 \mathrm{~min}$.

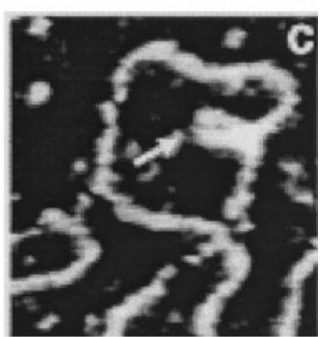

\section{$10 \mathrm{~min}$.}

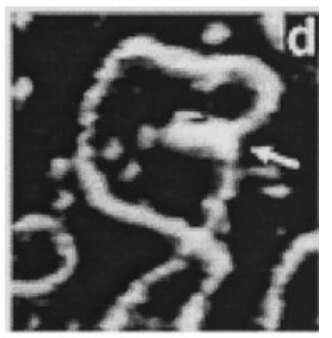

\section{$20 \mathrm{~min}$.}

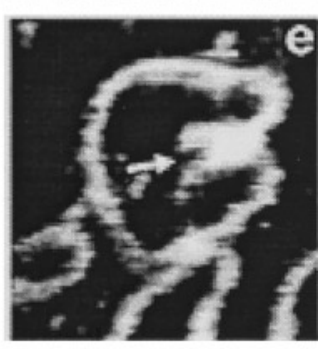

$34 \mathrm{~min}$.

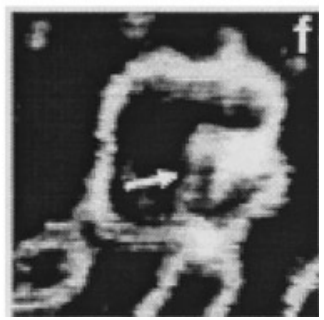

$40 \mathrm{~min}$.

Figure 3. AFM images of non-dried samples of pUC8F14C plasmid DNA taken by scanning in TE buffer. A and B are two consecutive scans over $\sim 500 \mu \mathrm{m} \times 500 \mu \mathrm{m}$ area. The molecules with three different conformations of the cruciform are indicated with arrows and numbered. The time difference between images A and B is ten minutes. C, The cruciforms in motion: consecutive scans of the fragments of molecules 1, 2 and 3 zoomed around only one cruciform; the time is indicated on the right. 
the cruciform (e.g. Figure 1B). We called this geometry of the cruciform extended conformation. However, more often we observed another orientation of the hairpin arms with rather acute angles between them (e.g. Figure 1C). The main DNA strands for this conformation of the cruciform are severely bent. This is a compact X-conformation. This geometry, which is stabilized by cations, is very dynamic allowing the hairpin arms to adopt almost parallel orientation. Extended conformation is less mobile and the hairpin arms prefer an orthogonal orientation. The supercoil-stabilized cruciform may adopt these two different conformations in solution as well. However, there may be an alternative interpretation of the data, that a continuum of conformations of the cruciform exists in solution and the two types of structures observed with the AFM are a consequence of deposition of a complex 3D-structure of the cruciform onto a plane. The geometry of immobilized cruciform may depend on virtual orientation of the molecule relative to the surface before the deposition, so the two structures observed would correspond to two different projections of the cruciform on the plane. There may also be a selection process that would lead to preferential binding to the surface of the cruciform in particular orientations of the arms. A close look at characteristics of the surface may help differentiate between these two alternatives.

Throughout this work we used AP-mica as a substrate for AFM studies. The AP-mica has specific surface characteristics that should be considered in understanding the behavior of DNA molecules and the cruciforms in particular at the surface-liquid interface and their interaction with the surface. As discussed in our previous publications (Lyubchenko et al., 1992, 1993b, 1995; Lyubchenko \& Shlyakhtenko, 1997), AP-mica is a positively charged surface with uniform and comparatively low surface charge density. There are three main consequences of these characteristics of AP-mica. First, the immobilization of DNA on APmica is due to electrostatic interactions between protonated amino groups of the substrate and the negatively charged DNA backbone. Second, due to uniform distribution of the charge on AP-mica there is no preferred orientation of DNA strands while they approach and bind to the surface. Third, owing to low surface charge density of AP-mica the geometry of immobilized supercoiled DNA molecules is close to two-dimensional projections of unperturbed DNA molecules onto a plane (Lyubchenko \& Shlyakhtenko, 1997). These features of AP-mica were crucial for unambiguous demonstration of the effect of ionic strength on the geometry of supercoiled DNA. Moreover, we also found that the shape of supercoiled DNA obtained at low and high ionic conditions are strikingly similar to computer simulated results obtained for supercoiled DNA molecules of similar lengths (Vologodskii \& Cozzarelli, 1994, 1996). These features of AP-mica are important prerequisites for application of this technique to AFM studies on the structure of supercoil-stabilized cruciforms.

Now we consider the adsorption onto AP-mica of the cruciform with a random orientation of the arms. Due to electrostatic character of DNA-surface interaction both arms (of the same length) should bind to the surface with the same efficiency. In addition, owing to the uniform distribution of aminopropyl groups on AP-mica, there should be no selectivity on binding of the arms with specific relative orientation. So all types of the cruciform conformations should be present on the surface. However, because of flatness of the AP-mica surface the conformations of the cruciform that are nearly planar should have higher binding affinities than non-flat ones. This selectivity effect depends on the strength of the arm-surface interaction which is a function of ionic strength. At low ionic strength, electrostatic interaction may be sufficient to hold the cruciform with X-type orientation of the arms despite its unfavorable geometry (at least one of the arms looks away from the surface). An increase of ionic strength will weaken the interaction between the arms, such that, at specific salt concentration, the forces between the arms and the surface may be insufficient to hold the cruciform with unfavorable X-type conformations. At such ionic conditions only conformations with a high number of contacts between the surface and the cruciform remain stable. From that point of view extended flat conformations of the cruciform rather than non-flat X-type conformations should have high affinity to the substrate. In other words, the increase of the salt concentration should lead to the increase of the ratio of extended conformations of the cruciform. So there are two main consequences of the alternative model. First, the cruciforms with random orientations of the arms should be observed with AFM. Second, the ratio of extended conformations should increase as ionic strength increases. We will show below that our AFM observations do not support these expectations.

First, not all orientations of the hairpin arms have been observed. For example, X-conformations with antiparallel orientation of the arms relative to the main DNA strands (see Figure 4) are very rare events (see p. 64). A more striking example is the geometry of the cruciform in extended conformations. In the majority of cases the hairpin arms tend to be perpendicular to the main strand (Figure 1). Second, the dependence of the ratio of extended conformations as a function of ionic strength is opposite to that expected from the alternative model: the number of cruciforms in extended conformations drops considerably at elevated salt conditions.

At the same time, both sets of evidence favor our interpretation, namely that these two types of conformations of the cruciform, the compact X-type structure and an extended conformation, exist in solution. It is important that this interpretation as well as the stabilization of X-type conformation of the cruciform in high salt solutions is in 


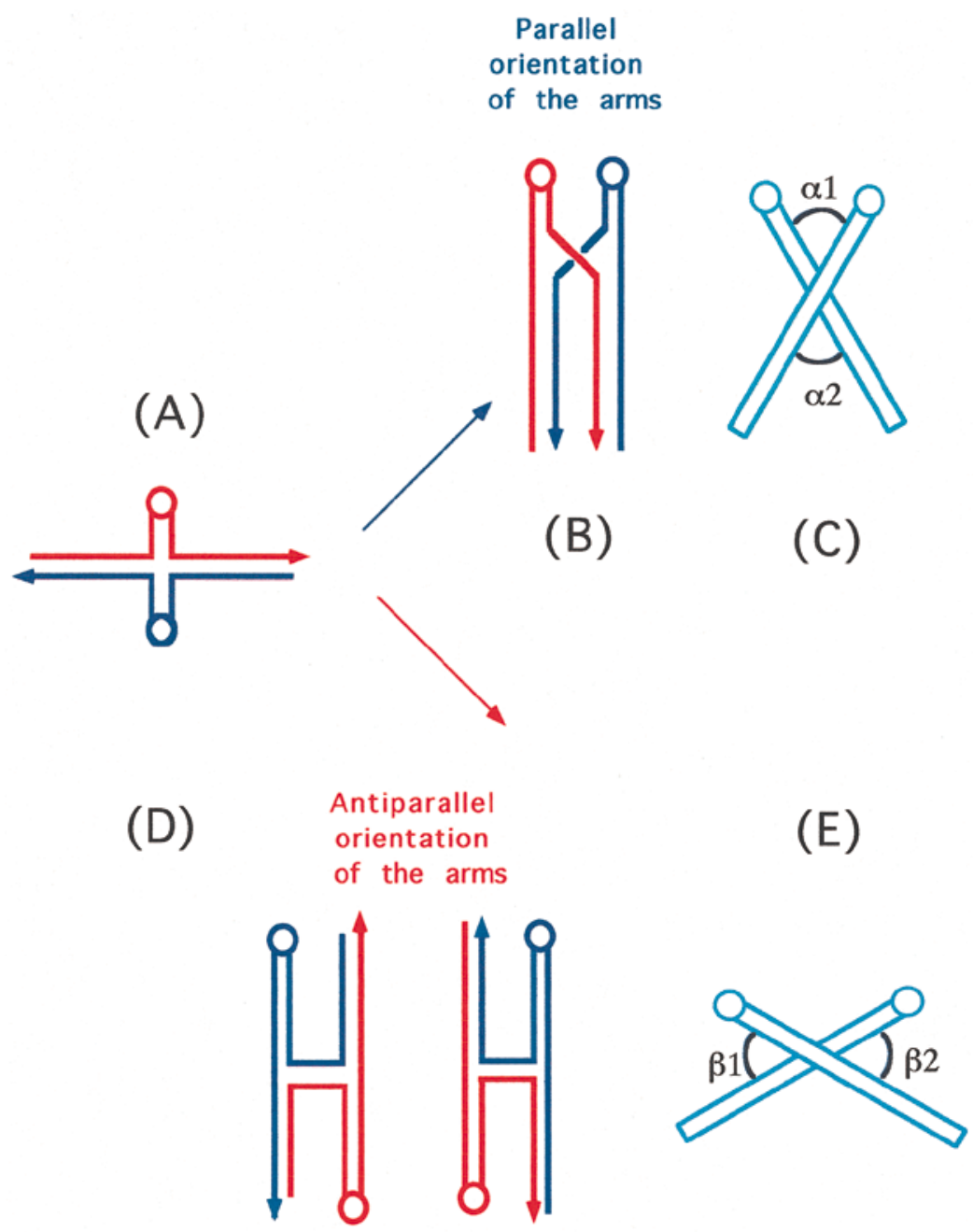

Figure 4. The cruciform and two different conformations of recombination DNA intermediates with parallel (B) and antiparallel (D) orientation of exchanging strands. The schemes of the cruciform in X-type conformation found here $(B, C)$ and the conformation of immobile four-way junctions (D, E) are drawn according to Seeman \& Kallenbach (1994).

accord with the data obtained for a rather similar system, immobile four-way junctions (Cooper \& Hagerman, 1987; Eis \& Millar, 1993; Lilley \& Clegg, 1993; Seeman \& Kallenbach, 1994; Lilley, 1997; Miick et al., 1997). Moreover, the AFM data indicate that the X-conformation has a strong bend in the main DNA strand, and this AFM observation is in remarkable coincidence with early gel retardation data (Gough \& Lilley, 1985; Zheng \& Sinden, 1988).

It is important to mention, however, the structures observed with the AFM are projections on the plane of a non-flat three-dimensional cruciform which may be distorted during the deposition onto the surface. In addition, it is formally possible that projections of different 3D-structures may be very similar and indistinguishable from the AFM, therefore other conformations of cruciforms in addition to the extended and the $\mathrm{X}$-conformation may exist in solution. It is also likely that the X-conformation is indeed a family of conformers with rather close energies, so transition between these structures can occur in solution at ambient conditions. Experimental evidence for the existence of a set different conformations of immobile four-way junctions was obtained recently by Miick et al. (1997).

\section{The dynamics of supercoil- stabilized cruciforms}

An interesting feature of the cruciforms emerges from their localization in the supercoil. As seen in Figure 2, the X-type cruciforms tend to be localized at the loops of the plectonemic superhelix. As shown earlier, intrinsically bent DNA regions prefer an apical localization in supercoiled DNA (Laundon \& Griffith, 1988). Our data suggest that cruciforms, like bent DNA sequences, are elements that can dictate a spatial geometry to a DNA superhelix. The compact X-type structure stabilized by cations was observed for immobile four-way junctions (Cooper \& Hagerman, 1987; Lilley \& Clegg, 1993; Seeman \& Kallenbach, 1994; Lilley, 
1997) and seems electrostatically reasonable at sites where DNA molecules make a U-turn.

We also found that cruciforms are dynamic structures. According to the data obtained for dried samples, the mean value for the angle between the arms of the X-type cruciform is $\sim 70^{\circ}$ and the standard deviation for this parameter is ca. $50 \%$. High mobility of the cruciform is also similar to that of immobile four-way junctions studied by different methods in solution (Cooper \& Hagerman, 1987; Eis \& Millar, 1993; Lilley, 1997; Miick et al., 1997). However, the AFM allowed us to obtain both, the numbers for the mean value of the angle between the arms and the variability of this parameter. The angles between the hairpin arms are very close to that for between the main strands which is demonstrated by statistical analysis (Table 1) and is seen in Figures 1 and 2: the pairwise values of the hairpin arm-main DNA strand angles in one molecule are very similar. In most cases these inter-arm angles for one molecule are almost identical, indicating that the arms move in a coordinated manner. It is reasonable to suggest that there is an interaction (stacking) between the cruciform helices at the base of the four-way junction. This assumption is in accord with data on chemical probing of cruciforms which indicates that the bases at the junction are much less reactive than those at the hairpin loops (Voloshin et al., 1989). At the same time, our experiments in liquid (Figure 3) show that the interaction between the arms is not strong enough to prevent the movement of the hairpin arms if long main arms are fixed at the surface. In this regard, comparatively low mobility of extended conformations of the cruciform seems quite surprising. We have found that the hairpin arms prefer an orthogonal orientation, so acute angles between the hairpin arms and the main strands are $\sim 80^{\circ}$ and variability of this parameter is ca. $15 \%$. These data obtained for dried samples are consistent with our observations performed in solution (Figure 3). Fully extended conformation of the cruciform with $\sim 90^{\circ}$ between the adjacent arms is a highly symmetric structure, which can be thermodynamically quite stable. Note in this regard that favorable conditions for observation of extended conformations of the cruciform is low ionic strength. So electrostatic repulsion of the nucleotides at the joint and the arms themselves may be two important factors that stabilize the fully extended configuration and thus limit its mobility. We also cannot exclude that fully extended configuration of the cruciform because of its flatness has high affinity to the surface, so this selectivity may lead to an elevated proportion of this specific conformation of the cruciform on the surface in comparison with their population in solution.

An alternative explanation for the high variability of the inter-arm angles for X-type conformation must be also considered. That is, in solution the cruciform adopts a set of interchangable conformations of the X-type family differing in the inter- arm angles. Each conformer has a mobility comparable with that of the extended conformation, but two-dimensional projection of the entire family would give a broad distribution of the angles that could be interpreted as a high mobility of one $\mathrm{X}$-conformation. However, in the experiments in liquid spontaneous movement of the hairpin arms was observed over a very broad range (Figure 3), thus the barrier between these conformations must be so low that it can be overcome by a thermal motion of the arms. Therefore, from this point of view, the X-type conformers are indistinguishable and unification of them under one X-type conformation is a rather natural first approximation.

\section{The conformation of cruciforms and its relation to DNA recombination and regulation of DNA replication and transcription}

As mentioned above, the supercoil-induced cruciform can be considered a putative model of the Holliday junction. First, the symmetry of the sequence of the cruciform by definition permits the branch migration. Second, genomic DNA is organized in topological domains with unrestrained supercoiling (Sinden, 1994) and topoisomerases are involved at the steps of homologous pairing and DNA strand transfer (Kowalczykowski, 1994), suggesting that DNA supercoiling is an important factor for the DNA recombination.

Currently existing models for recombination of DNA are shown schematically in Figure 4. In addition to extended conformation of the cruciform (A) two different orientations of the arms ((B) and (D)) that correspond respectively to parallel (B) and antiparallel (D) orientations of exchanging arms are possible (Sigal \& Alberts, 1972; Seeman \& Kallenbach, 1994; Sobell, 1974). The model (B) with a parallel orientation of exchanging strands suggested by Sigal \& Alberts (1972) easily explains the strand exchange by a branch migration mechanism. According to Table 1 (column 1), the interarm angles 1 and 2 are rather acute (see Figure $4(\mathrm{C})$ ). The molecules with obtuse angles 1 and 2, as shown in Figure 4(E), were very rare events in the AFM images, and molecules with closer distances between the hairpins and the DNA strands were not observed. So the AFM data are consistent with the model (B) of geometry of cruciforms which is equivalent to the Holliday junction with a parallel orientation of exchanging strands. It is surprising that a seemingly similar type of structure, an immobile four-way junction, can adopt the geometry (D) which corresponds to antiparallel strand orientation (Figure 4(E); Cooper \& Hagerman, 1987; Lilley \& Clegg, 1993; Seeman \& Kallenbach, 1994; Lilley, 1997). Although antiparallel and parallel states of immobile junctions are thermodynamically very close ( Lu et al., 1991) and the barrier between the two conformations is not high (Srinivasan \& Olson, 1994), only an antiparallel strand orientation in immobile junctions has been observed (Cooper \& Hagerman, 1987; Lilley 
\& Clegg, 1993; Seeman \& Kallenbach, 1994; Lilley, 1997; Eis \& Millar, 1993). The migration of the crossover site of antiparallel conformation of the junction requires considerable conformational changes, but once the helical arms are aligned in a parallel manner, the branch migration proceeds with relatively little energetic costs (Srinivasan \& Olson, 1994). The nature of a supercoiled topological domain and the propensity for bent DNAs such as a cruciform (Gough \& Lilley, 1985; Zheng \& Sinden, 1988) to orient themselves at the tip of a loop (Figure 2) strongly favor the parallel geometry. Probably, the constraints placed on the structure of the Holliday junction by topology and supercoiling outweigh a small effect of an energy difference in the parallel and antiparallel structures of four-way junctions. Thus, DNA supercoiling and the topological domain organization in chromosomes may dictate the structure of a Holliday junction.

A number of other biological implications follow from the features described for cruciforms. The ability of cruciforms and four-way DNA junctions (Cooper \& Hagerman, 1987; Lilley \& Clegg, 1993; Seeman \& Kallenbach, 1994; Lilley, 1997; Srinivasan \& Olson, 1994; Eis \& Millar, 1993; Petrillo et al., 1988) to adopt different conformations might be functionally important. These DNA structures are the targets for different types of proteins such as recombination enzymes (Parsons et al., 1995; Yu et al., 1997), topoisomerases (Roca et al., 1993), and architectural proteins such as HMG1 and histones H1 and H5 (Timsit \& Moras, 1996), so that the conformational flexibility of DNA is an important factor for accommodating such a plethora of proteins. For example, the variability of the cleavage patterns of the same fourway junctions for resolving enzymes from different species (Lilley, 1997) is easily explained by a dynamic character of the four-way junction. Another example is the interaction of four-way junctions with the E. coli RuvA, B and C (Parsons et al., 1995; Yu et al., 1997). An extended conformation of the four-way junction (Figure 4(A)) is the target for RuvA and RuvB proteins at the initial step of the recombination process, but the final step, resolving the junction with $\mathrm{RuvC}$, requires a compact X-type conformation (Lilley \& Clegg, 1993).

There are other known examples of the role of cruciforms in biology. The formation of supercoilinduced cruciforms changes the level of unrestrained torsional stress in topological domains of the genome, which could influence gene regulation or replication. The ability of cruciforms to move may facilitate their interaction with specific proteins, and this may be important for regulation of DNA replication (Noirot et al., 1990) and transcription (Glucksmann et al., 1992), where roles for cruciforms have been demonstrated. AFM has already been successfully applied to structural studies of complexes of linear DNA with a number of nucleoprotein complexes (Bustamante et al., 1994;
Hansma \& Hoh 1994; Lyubchenko et al., 1995). Now that the structural information is available from AFM studies of supercoiled DNA, the technique might be applied to complexes of supercoiled DNA with the key proteins of transcription, replication and recombination.

\section{Materials and Methods}

\section{DNA preparation}

Plasmid pUC8F14C with a $106 \mathrm{bp}$ inverted repeat (Sinden, 1994) was grown in E. coli strain HB101 and isolated by cell lysis with $1 \%(\mathrm{v} / \mathrm{v})$ Brij $58,0.4 \%(\mathrm{w} / \mathrm{v})$ deoxycholate, followed by $\mathrm{CsCl}$ centrifugation. Topoisomers of particular superhelical densities were generated by incubating supercoiled plasmid with a topoisomerase extract from HeLa cells in the presence of different concentrations of ethidium bromide (Kochel \& Sinden, 1988). The superhelical densities of closed circular molecules (about $80 \%$ of DNA molecules in topoisomer fractions) were estimated by $1.5 \%(\mathrm{w} / \mathrm{v})$ agarose gel electrophoresis in the presence of 3,9 and $25 \mu \mathrm{g} / \mathrm{ml}$ chloroquine phosphate. To determine the superhelical density required for cruciform formation, a mixture of topoisomers (about $0.25 \mu \mathrm{g}$ each fraction) was separated in a $1.5 \%$ agarose two-dimensional gel $(15 \mathrm{~cm} \times 15 \mathrm{~cm})$ at $25^{\circ} \mathrm{C}, 4 \mathrm{~V} / \mathrm{cm}$ using Topo TAE buffer (40 mM Tris$\mathrm{HCl}, 5 \mathrm{mM}$ sodium acetate, $1 \mathrm{mM}$ EDTA, $\mathrm{pH}$ 8.3) for the first dimension and the same buffer containing $30 \mu \mathrm{g} / \mathrm{ml}$ chloroquine phosphate for the second dimension.

\section{AFM procedure}

Throughout this work only mica functionalized with 3-aminopropyltriethoxy silane (AP-mica) was used as a substrate (Lyubchenko et al., 1992, 1993a,b, 1995; Lyubchenko \& Shlyakhtenko, 1997). For imaging in air, $10 \mu \mathrm{l}$ of the DNA $(0.5 \mu \mathrm{g} / \mathrm{ml})$ in TE buffer was placed onto pieces of AP-mica for two minutes, thoroughly rinsed with deionized water (ModuPure Plus, Continental Water System Corp., San Antonio, TX) and argondried. NanoProbe TESP (Digital Instruments, Inc., Santa Barbara, CA) tips were used for imaging in air. The typical tapping frequency was 240 to $280 \mathrm{kHz}$; scanning rate was $1.97 \mathrm{~Hz}$. For imaging in liquid, $\mathrm{Si}_{3} \mathrm{~N}_{4}, 100 \mu \mathrm{m}$ long, NanoProbe tips mounted on a glass tip holder for tapping mode (Digital Instruments, Inc., Santa Barbara, CA) were used. The tip was manually approached to the AP-mica surface mounted on the sample stage on the microscope until the distance between the tip and the surface was 20 to $50 \mu \mathrm{m}$. The DNA solution $(25$ to $50 \mu \mathrm{l})$ was injected into a gap between the mica surface and the tip holder and the tip was brought into a tapping range under control of the instrument. The scanning parameters were as follows: frequency 8 to $9 \mathrm{kHz}$, scanning rate $1.97 \mathrm{~Hz}$. The images were taken in the topographic mode. A NanoScope III MultiMode system (Digital Instruments, Inc., Santa Barbara, CA) operated in tapping mode was used for these experiments.

\section{Analysis of the AFM data}

$\mathrm{NIH}$ image 1.60 software was used for measurements of the angles between the arms. Statistical analysis of the data was performed by using Kaleidagraph 3.0.1. software. The number of the molecules used for measurements of the angles for X-type conformation of the 
cruciforms are indicated in Table 1 (the last column). Similar dataset (31 molecules) was used for measuring the angles between arms for extended conformations of the cruciform.

\section{Acknowledgments}

This work was supported in part by grants GM 54991 from NIH and Digital Instruments, Inc. We are grateful to G. Manning, W. Olson, N Seeman and V. Zhurkin for reading the manuscript and valuable comments.

\section{References}

Binnig, G., Quate, C. F. \& Gerber, C. H. (1986). Atomic force microscope. Phys. Rev. Letters, 56, 930-933.

Bustamante, C., Erie, D. A. \& Keller, D. (1994). Biochemical and structural applications of scanning force microscopy. Curr. Opin. Struct. Biol. 3, 750760.

Cooper, J. P. \& Hagerman, P. J. (1987). Gel electrophoretic analysis of the geometry of a four-way junction. J. Mol. Biol. 198, 711-719.

Eis, P. S. \& Millar, D. P. (1993). Conformational distributions of a four-way DNA junction revealed by time-resolved fluorescence resonance energy transfer. Biochemistry, 32, 13852-13860.

Gierer, A. (1966). Model for DNA and protein interaction and the function of the operator. Nature, 212, 1480-1481.

Glucksmann, M. A., Markiewicz, P., Malone, C. \& Rothman-Denes, L. R. (1992). Specific sequences and a hairpin structure in the template strand are required for $\mathrm{N} 4$ virion RNA polymerase promoter recognition. Cell, 70, 491-500.

Gough, G. W. \& Lilley, D. M. J. (1985). DNA bending induced by cruciform formation. Nature, 313, 154156.

Hansma, H. G. \& Hoh, J. (1994). Biomolecular imaging with the atomic force microscopy. Annu. Rev. Biophys. Biochem. Struct. 23, 115-139.

Kochel, T. \& Sinden, R. R. (1988). Analysis of trimethylpsoralen photoreactivity to Z-DNA provides a general in vivo assay for Z-DNA: analysis of the hypersensitivity by (GT)n B-Z junctions. Biotechniques, 6, 532-543.

Kornberg, A. \& Baker, T. A. (1992). DNA Replication, Freeman, New York.

Kowalczykowski, S. C. (1994). In vitro reconstituion of homologous recombination reactions. Experimentia, 50, 204-215.

Laundon, C. H. \& Griffith, J. D. (1988). Curved helix segments can uniquely orient the topology of supertwisted DNA. Cell, 52, 545-549.

Lilley, D. M. J. (1997). All change at Holliday junction. Proc. Natl Acad. Sci. USA, 94, 9513-9515.

Lilley, D. M. J. \& Clegg, R. M. (1993). Parallel and antiparallel Holliday junctions differ in structure and stability. Annu. Rev. Biophys. Biomol. Struct. 22, 299328.

Lilley, D. M. J. \& Kemper, B. (1984). Cruciform-resolvase interactions in supercoiled DNA. Cell, 36, 413-422.

Lu, M., Guo, Q., Seeman, N. C. \& Kallenbach, . N. R. (1991). Parallel and antiparallel Holliday junctions differ in structure and stability. J. Mol. Biol. 221, 1419-1432.
Lyubchenko, Y. L. \& Shlyakhtenko, L. S. (1997). Visualization of supercoiled DNA with atomic force microscopy. Proc. Natl Acad. Sci. USA, 94, 496501.

Lyubchenko, Y. L., Gall, A. A., Shlyakhtenko, L. S., Harrington, R. E., Jacobs, B. L., Oden, P. I. \& Lindsay, S. M. (1992). Atomic force microscopy imaging of dsDNA and RNA. J. Biomol. Struct. Dynam. 10, 589-606.

Lyubchenko, Y. L., Oden, P. I., Lampner, D. \& Lindsay, S. M. (1993a). Atomic Force Microscopy of DNA and bacteriophage in air, water and propanol: role of adhesion forces. Nucl. Acids Res. 21, 1117-1123.

Lyubchenko, Y. L., Shlyakhtenko, L. S., Harrington, R. E., Oden, P. I. \& Lindsay, S. M. (1993b). Atomic force microscopy of long DNA: imaging in air and under water. Proc. Natl Acad. Sci. USA, 90, 21372140.

Lyubchenko, Y. L., Jacobs, B. L., Lindsay, S. M. \& Stasiak, A. (1995). Atomic force microscopy of nucleoprotein complexes. Scann. Microsc. 9, 705727.

McClellan, J. A. \& Lilley, D. M. J. (1987). A two-state conformational equilibrium for alternating (A-T) $n$ sequences in negatively supercoiled DNA. J. Mol. Biol. 197, 707-721.

Miick, S. M., Fee, R. S., Millar, D. P. \& Chazin, W. J. (1997). Crossover isomer bias is the primary sequence-dependent property of immobilized Holliday junctions. Proc. Natl Acad. Sci. USA, 94, 90809084.

Mizuuchi, K., Mizuuchi, M. \& Gellert, M. (1982). Cruciform structures in palindromic DNA are favored by DNA supercoiling. J. Mol. Biol. 156, 229-243.

Noirot, P., Bargonetti, J. \& Novick, R. P. (1990). Initiation of rolling-circle replication in pT181 plasmid: initiator protein enhances cruciform extrusion at the origin. Proc. Natl Acad. Sci. USA, 87, 85608564.

Palecek, E. (1992). Probing of DNA structure in cells with osmium tetraoxide-2. 2'-bipyridine. Methods Enzymol. 212, 305-318.

Parsons, C. A., Stasiak, A., Bennett, R. J. \& West, S. C. (1995). Structure of a multisubunit complex that promotes DNA branch migration. Nature, 374, 375378.

Petrillo, M. L., Newton, C. J., Cunningham, R. P., Ma, R. I., Kallenbach, N. R. \& Seeman, N. C. (1988). Ligation and flexibility of four-arm DNA junctions. Biopolymers, 27, 1337-1352.

Roca, J., Berger, J. M. \& Wang, J. C. (1993). On the simultaneous binding of eukaryotic DNA topoisomerase II to a pair of double-stranded DNA helices. J. Biol. Chem. 268, 14250-14255.

Seeman, N. C. \& Kallenbach, N. R. (1994). DNA branched junctions. Annu. Rev. Biophys. Biomol. Struct. 23, 53-86.

Sigal, N. \& Alberts, B. (1972). Genetic recombination: the nature of a crossed strand-exchange between two homologous DNA molecules. J. Mol. Biol. 71, 789-793.

Sinden, R. R. (1994). DNA Structure and Function, pp. 134-178, Academic Press, San Diego.

Sinden, R. R., Broyles, S. S. \& Pettijohn, D. E. (1983). Perfect palindromic lac operator DNA sequence exists as a stable cruciform structure in supercoiled DNA in vitro but not in vivo. Proc. Natl Acad. Sci. USA, 80, 1797-1801. 
Sobell, H. M. (1974). Concerning the stereochemistry of strand equivalence in genetic recombination. In Mechanisms in Recombination (Grell, R. F., ed.), pp. 433-438, Plenum Publishing Corp., New York.

Srinivasan, A. R. \& Olson, V. K. (1994). Computer models of DNA four-way junctions. Biochemistry, 33, 9389-9404.

Timsit, Y. \& Moras, D. (1996). Cruciform structures and functions. Qaurt. Rev. Biophys. 29, 279-307.

Vologodskii, A. V. (1992). Topology and Physics of Circular DNA, pp. 31-153, CRC Press, Boca Raton.

Vologodskii, A. V. \& Cozzarelli, N. R. (1994). Annu. Rev. Biophys. Biomol. Struct. 23, 609-643.
Vologodskii, A. V. \& Cozzarelli, N. R. (1996). Effect of supercoiling on the juxtaposition and relative orientation of DNA sites. Biophys. J. 70, 2548-2556.

Voloshin, O. N., Shlyakhtenko, L. S. \& Lyubchenko, Y. L. (1989). Localization of melted regions in supercoiled DNA. FEBS Letters, 243, 377-380.

Yu, X., West, S. C. \& Egelman, E. H. (1997). Structure and subunit composition of the RuvAB-Holliday junction complex. J. Mol. Biol. 266, 217-222.

Zheng, G. \& Sinden, R. R. (1988). Effect of base composition at the center of inverted repeated DNA sequences on cruciform transition in DNA. J. Biol. Chem. 263, 5356-5361.

Edited by I. Tinoco

(Received 21 November 1997; received in revised form 7 April 1998; accepted 8 April 1998) 